\title{
Cold cathode arc attachment: The importance of the high local pressure
}

\author{
Jean-Luc Meunier and Sylvain Coulombe \\ Plasma Technology Research Center, Chemical Engineering Department, McGill University, \\ Montréal, Québec, CANADA H3A 2B2

\begin{abstract}
This study is related to the fundamental aspects of the arc-cathode interactions in atmospheric and higher-pressure arc systems. The primary objective of this paper is to demonstrate that, even though the arc attachment on both refractory and non-refractory cathodes appear substantially different, they follow the same physics and therefore the same model can be applied to describe both situations. Indeed, it is shown that the two situations are actually representing the two extremes of a unique description; the self-sustaining operation of an arc discharge on a refractory (hot) cathode is achieved without the need of vaporization of the cathode surface while for non-refractory (cold) cathodes, a strong vaporization resulting in a pressure build up in the cathode region is necessary for self-sustained arc operation.
\end{abstract}

\section{INTRODUCTION}

The arc-cathode interactions have been the subject of a considerable interest since several years due mainly to the erosion problems that often impact on the industrial applications of thermal arc plasma technology. It has long been thought that the erosion is due to the impossibility for the cathode to dissipate the strong incoming heat flux from the arc column by conduction or radiation. Several designs have been reported for cold-cathode systems to enhance the heat dissipation by either increasing the water cooling efficiency of the cathode or by forcing the arc root to move by magnetic or gas dynamic means in order to reduce the local heat loads. However, cathode erosion is still observed. On the other hand, a few researchers brought the idea that the cathode erosion might actually be necessary for the self-sustaining operation of the arc discharge [1, 2]. In such a view of the arc-cathode interactions, one would understand that the erosion is related to the cathode ability to meet the necessary conditions for a self-sustaining operation. Practical experiences show that cathodes made of refractory materials such as tungsten meet these conditions without strong erosion while this is not the case with non-refractory materials such as copper. It is the aim of this study to show how the different cathode materials achieve these necessary conditions as a function of their refractory character.

\section{BACKGROUND ON ARC-CATHODE INTERACTIONS}

\section{Basic Knowledge}

Cathodes of high pressure arc systems are generally classified as thermionic or non-thermionic emitters depending on their ability to sustain a strong thermionic emission of electrons without strong melting or vaporization. The parameter best-suited to describe this 'ability' is the ratio of the flux of vaporized atoms (Langmuir equation) to the flux of thermionic electrons (Richardson-Dushman equation) defined as: 


$$
\frac{e \Gamma_{m p}}{j_{R D}}=\frac{p / 4\left(m k T_{s} / 3\right)^{1 / 2}}{4 \pi m m_{e} k^{2} T_{s}^{2} \exp \left(-e \phi / k T_{s}\right) / h^{3}}
$$

where $T_{\mathrm{s}}$ is the cathode surface temperature while $p, m$ and $\phi$ are respectively, the vapor pressure at temperature $T_{\mathrm{s}}$, the atomic mass and the work function of the cathode material. The lower is the ratio $e \Gamma_{\mathrm{Vap}} / j_{\mathrm{RD}}$, the better-suited is the cathode material for thermionic operation. This ratio is plotted in Fig. 1 as a function of the cathode surface temperature for several cathode materials. Note that this ratio does not provide for an accurate picture of the conditions prevailing in the cathode region since the effects due to the electric field on the electron emission conditions (Schottky effect, field emission) are not taken into account. It however provides for a representative picture of the effect of the cathode material nature. All the curves are plotted until the normal boiling point of the cathode material is reached.

One can define the thermionic cathode materials as those for which $e \Gamma_{\mathrm{ved}} / j_{\mathrm{RD}}<1$, and the non-thermionic materials as those for which $e \Gamma_{\mathrm{vap}} / j_{\mathrm{RD}}>1$. Two important remarks can be made from an brief analysis of Fig.1: 1) Obviously the thermionic emitters are made of refractory materials. 2) As the temperature increases, the 'ability' for the non-refractory cathode materials to operate in the thermionic regime increases. However, for all the non-refractory materials considered, more than one atom is emitted per electron extracted from the cathode surface and this, even when the cathode surface is boiling. This observation shows the nature of the erosion problems encountered with non-refractory cathodes, but an even more serious problem is hidden here: according to modeling predictions (see [5] for a complete description of the model), the arc discharge is not self-sustaining for all the non-refractory cathode materials and this, even when the cathode surface temperatures reach their respective normal boiling point.

\section{Definition of the Problem of}

\section{Self-sustaining Operation}

The principal role of the cathode is to provide a sufficient flux of electrons to maintain the electric arc discharge. These electrons (beam electrons) are emitted from the cathode under the combined actions of the high surface temperatures and electric fields maintained mainly by the ion flux bombarding the cathode surface and forming the cathode sheath. Once

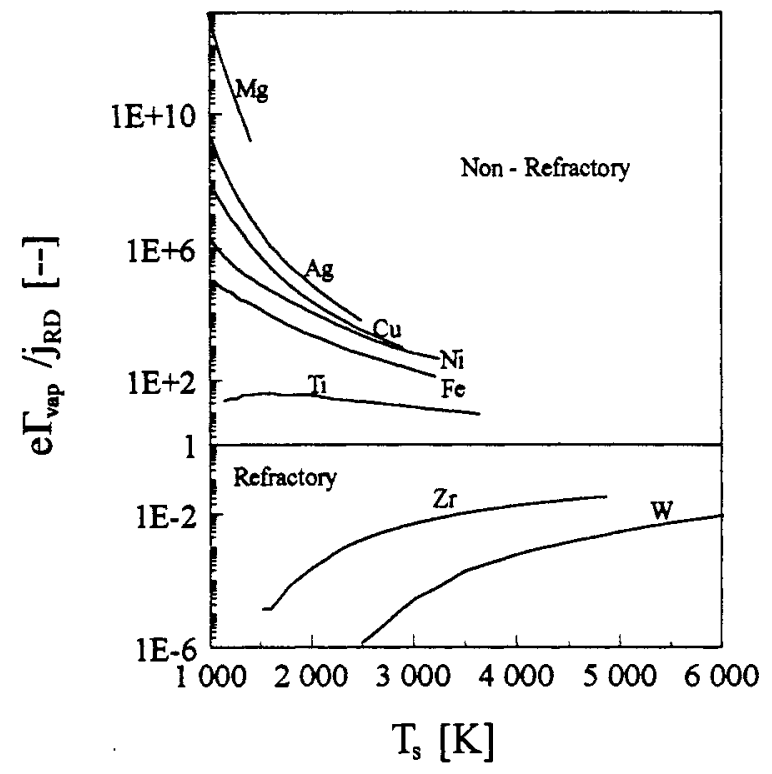

Fig. 1. Calculated ratio of the flux of vaporized atoms $\left(\Gamma_{\text {vap }}\right)$ to the flux of thermionic electrons $\left(j_{\mathrm{pD}} / e\right)$ as a function of the cathode surface temperature. emitted from the cathode, the electrons are accelerated through the cathode sheath without making collisions. Once the ionization zone is reached by these electrons, inelastic collisions with neutrals produce ions and result in the formation of an electrically-conducting channel between the two electrodes (arc column formation). According to this scenario, it is obvious that the self-sustaining operation of the arc discharge is possible only if the 
conditions prevailing in the cathode region are those favoring' a sufficient electron emission. It is on the mechanisms these conditions are achieved that refractory and non-refractory cathodes differ strongly.

The fraction $f_{b i}$ of the beam electrons (index $b$ ) that are left for ionization after collisions can be defined as:

$$
f_{b i}=\frac{v_{b i}}{v_{b i}+v_{b e}}=\frac{\sigma_{b i}}{\sigma_{b i}+\sigma_{b e}}<1
$$

where $i$ stands for ionization and $e$ for excitation, the $v$ 's are the frequencies of the collision processes and the $\sigma$ 's, the collision cross sections. Obviously, $f_{0 \mathrm{~b}}$ is less than unity. The maximum energy taken by the beam electrons before collisions corresponds to the cathode sheath voltage drop, and is therefore less than 10-20 eV for high pressure arcs. For this energy range, only single-ionization occurs upon electron impact [3]. This observation and the condition defined by equation (2) allow to define the most restraining criterion for the self-sustaining operation of an arc discharge maintained by an electron beam emitted from the cathode $\left(j_{T-F}\right)$ :

$$
\frac{j_{i o n}}{j_{T-F}}<1
$$

This criterion states that the flux of ions produced by ionization of the neutrals by the beam electrons cannot be higher that the flux of beam electron that generates it. According to the calculations of Zhou and Heberlein [4], the ratio $j_{\text {ion }} / j_{\mathrm{T} F}$ ranges from $\sim 0.25$ to 0.4 for a W cathode interacting with an atmospheric pressure argon arc [4]. With non-refractory $\mathrm{Cu}, \mathrm{Fe}$ or Ti cathodes, this ratio is much larger than unity even for surface temperatures up to the boiling point of the cathode material (based on our calculations). The impossibility to obtain the self-sustaining operation of the arc stands in the insufficient electron emission from the surface. In order to solve this problem, a new approach for the description of the arc attachment on such non-refractory cathode materials must be introduced.

\section{Concept of High Local Pressure}

As concluded by Guile [1], it seems that the self-sustaining operation of an high pressure arc on a nonrefractory cathode is possible only if some destruction of the cathode surface occurs. The presence of a high density of metallic vapors in the cathode region would have the effect of maintaining a high ion bombardment (high surface temperatures) and a high surface electric field (cathode sheath compression) which in turm, would allow a high current density of beam electrons $[5,6]$. In this scenario, the returning ions bombarding and heating the surface maintain the strong vaporization and a pressure build up in the cathode region. The ions are essentially the vaporized cathode material atoms having been ionized in the ionization zone and accelerated back towards the cathode by the cathode sheath voltage drop. Similar to the vacuum arc cathode spot [7], the cathode region of a high pressure arc on a non-refractory material is then composed of a high density metallic plasma formed by vaporization of the cathode. This picture fits well with the smooth transition in erosion rate and metal vapour expansion behavior in the transition between vacuum and atmospheric pressure arcs, this transition occuring essentially between 1 Torr and atmospheric pressure $[8,9]$. The conditions within the cathode region allowing a self-sustaining operation of the arc will be those satisfying the criterion defined with equation (3) and satisfying the energy balance at the cathode surface.

One of the consequences of such a pressure build up is that the ambient gas filling the chamber is plowed off the cathode region when a new cathode spot is ignited [10]. The plasma of the cathode region is therefore locally burning in pure metallic vapors at pressures possibly well above the ambient gas pressure. 
The physics lying behind the description of the arc attachment on non-refractory cathodes is the same as for refractory cathodes; only the medium in which the plasma of the cathode region is burning in is different. Therefore, a unique model for all the metallic elements can be defined.

\section{RESULTS AND DISCUSSION}

The physical model used to determine the conditions prevailing within the cathode region (described in details in [5]) has three independent parameters; respectively the cathode sheath voltage drop $V_{c}$, the metallic plasma pressure $p$ and the electron temperature $T_{e}$ at the edge of the cathode sheath. The cathode surface temperature $T_{s}$ is related to the local pressure $p$ via a vapor-pressure curve, i.e. $T_{s}=T_{s}(p)$. The current densities and heat fluxes to the cathode are calculated from these conditions. For the results presented in this study, $V_{\mathrm{c}}$ was set equal to $15 \mathrm{~V}$, and $T_{\mathrm{e}}$ allowed to vary between 1 and $2 \mathrm{eV}$ for the reasons discussed in [5].

The evolution of the self-sustaining criterion defined by equation (3) is plotted in Fig. 2 as a function of $p$ and $T_{\mathrm{e}}$ for a cathode spot plasma on a pure $\mathrm{Cu}$ cathode. It is shown that the arc discharge becomes selfsustaining for metallic plasma pressures exceeding $\sim 19 \mathrm{~atm}$. The current density at the cathode surface under these conditions is $3.5 \times 10^{9} \mathrm{~A} \mathrm{~m}^{-2}$. The minimum pressure for a self-sustaining operation depends strongly on the electron temperature since all the properties calculated by the model are strongly dependent on the electron density $n_{e}$, itself dependent on $T_{e}$. The electron emission from the cathode is considerably enhanced with respect to the vaporization rate as the local pressure increases since for sufficiently high pressures, strong thermo-field emission from the cathode surface occurs [5, 6]. Indeed for high values of $p$, both the electron and the ion densities are high. This results in an important ion bombardment on the cathode and high surface temperatures favoring electron emission. Also, the presence of a high density of charged particles in the cathode region results in an important compression of the cathode sheath and therefore, the establishment of high surface electric fields that also favor electron emission (Schottky effect and field emission). Finally, the presence of a high density of ions further enhance the electron emission due to important modification of the potential barrier for the elzctrons in the close vicinity of the cathode surface [11]. The local plasma pressure in the cathode region cannot take any arbitrary high value. As the electron emission from the cathode increases, the cathode cooling effect associated with this emission also increases (Nottingham effect, see Figure 10 in [5]). Therefore, a maximum pressure is reached above which the incoming heat flux from the plasma becomes insufficient to balance these electron emission energy losses. For a $\mathrm{Cu}$ cathode, this maximum pressure is around $70 \mathrm{~atm}$ corresponding to a maximum current

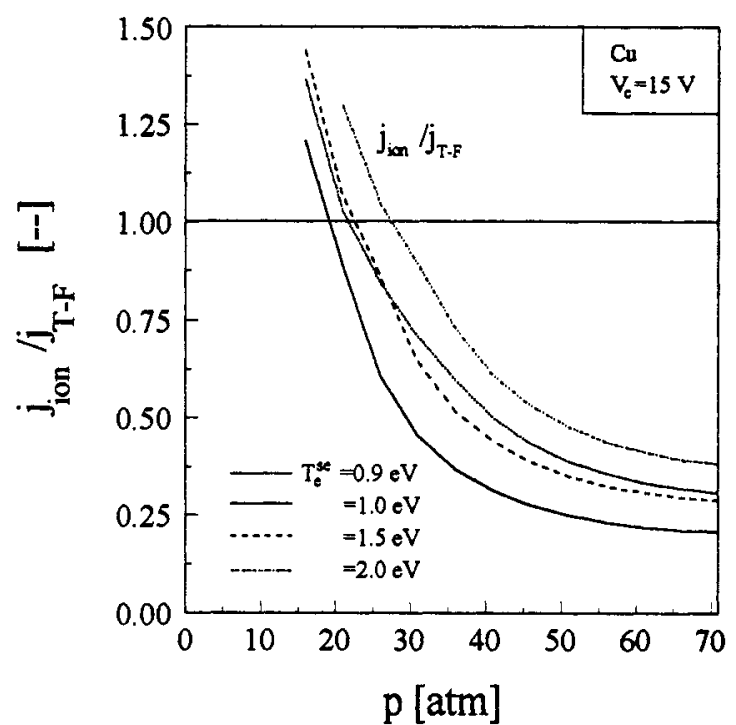

Fig. 2. Ratio $j_{\text {ion }} / j_{\mathrm{T}-\mathrm{F}}$ for a $\mathrm{Cu}$ cathode spot plasma as a function of the metallic vapor pressure and electron temperature. 
density of $5 \times 10^{10} \mathrm{~A} \mathrm{~m}^{-2}$.

It is now interesting to plot again the ratio of the flux of vaporized atoms $\left(\Gamma_{\text {vep }}\right)$ to the flux of emitted electrons of Fig. 1, but now using the actual conditions of thermofield emission prevailing in the cathode region of cold-cathode systems. The factor $j_{R D}$ in equation (1) is thus replaced by $j_{\text {T.F }}$, the thermo-field emission current density. The ratio $e \Gamma_{\mathrm{ved}} / j_{\mathrm{T} \cdot \mathrm{F}}$ is plotted in Fig. 3 . Surprisingly for the self-sustaining operating conditions given by equation (3) and Figure 2 ( $p>19 \mathrm{~atm}$ ), non-refractory cathodes behave in a similar way as refractory cathodes: they both show ratios of the flux of vaporized atoms to the flux of emitted electrons that is lower than unity. This clearly shows that no special distinction need to be made on the nature of the cathode material when attempting to describe the arc attachment. Tungsten with its boiling point at $5830 \mathrm{~K}$ corresponds to the limiting behavior for which the ionization of the ambient gas atoms ( $\mathrm{Ar}$ for instance) is sufficient to promote the necessary conditions for a self-sustaining arc discharge. Copper represents the other limiting behavior for which a pressure build up in the cathode region and ionization of the vaporized atoms are necessary to achieve the required electron emission conditions.

This brief analysis of the two limiting behaviors suggests that a continuous transition should be observed for metallic elements showing intermediate properties such as $\mathrm{Fe}$ and $\mathrm{Ti}$. This is shown in Fig. 4 where the ranges of self-sustaining plasma pressures obtained from the model [5] for $\mathrm{Cu}, \mathrm{Fe}$ and $\mathrm{Ti}$ are plotted as a function of the respective boiling temperatures. The range of metallic pressures corresponding to the experimental estimation of the cathode surface temperature for a W cathode exposed to an atmospheric pressure Ar arc [12] is also plotted in Fig. 4 to show the continuous

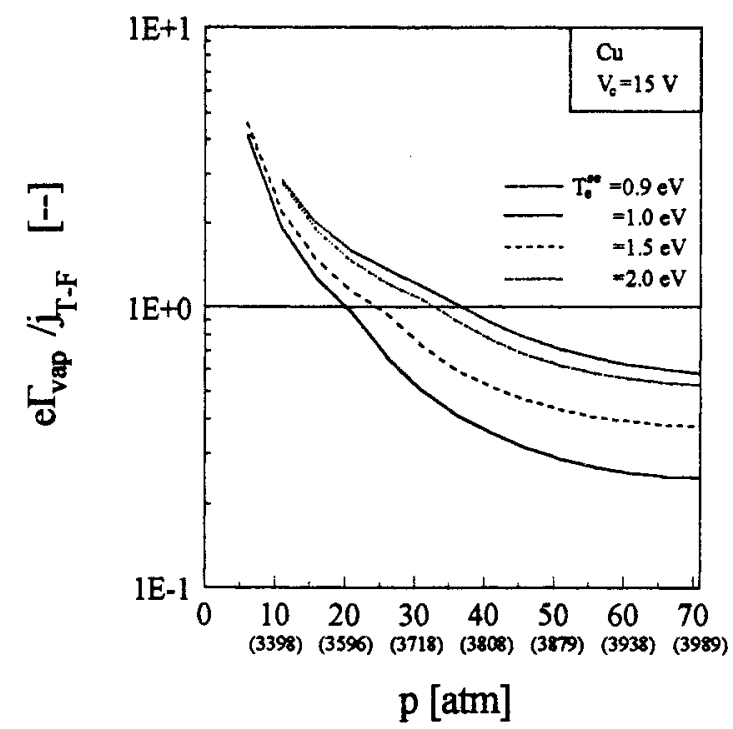

Fig. 3. Ratio of the flux of vaporized atoms $\left(\Gamma_{\mathrm{nmp}}\right)$ to the actual flux of emitted electrons $\left(j_{T-F} / e\right)$ as a function of the metallic vapor pressure and electron temperature (the corresponding cathode surface temperatures $(K)$ are in parentheses).

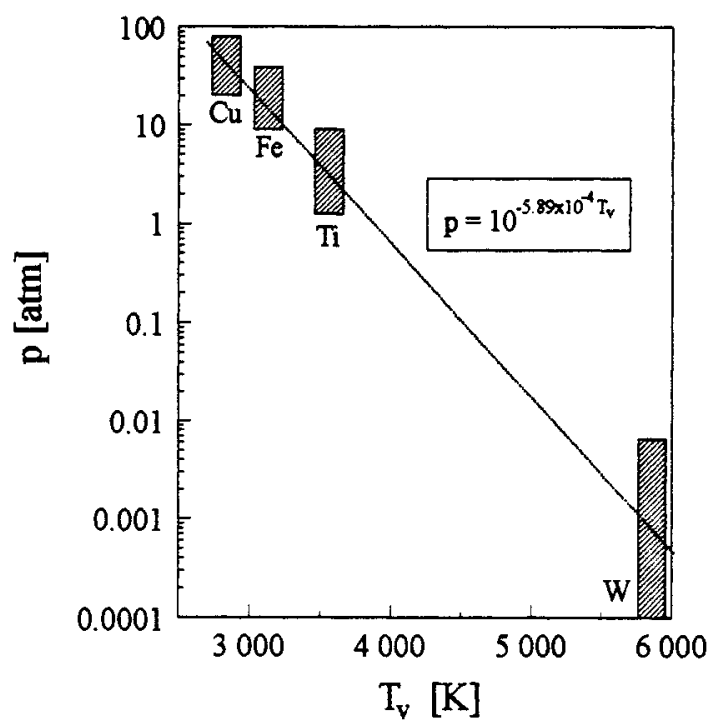

Fig. 4. Range of metallic vapor pressures in the cathode region needed for a self-sustaining operation of the arc discharge for several cathode materials as a function of the respective boiling temperatures. 
4 to show the continuous evolution from $\mathrm{Cu}$ to $\mathrm{W}$. The present model did not allow to calculate such operating conditions for $\mathrm{W}$. In such systems for which the metallic vapor pressure is below the ambient gas pressure, the cathode surface temperature cannot anymore be simply related to the pressure via a vaporpressure curve. One needs to couple the cathode sheath model to a beat transfer model of the cathode in order to calculate the surface temperature. This is however a simple implementation to the cathode sheath model described in [5]. Results presented in Fig. 4 show that a continuous transition occurs between a regime of self-sustaining arc attachment necessitating a pressure build up in the cathode region (e.g. $\mathrm{Cu}$ ) to a regime not necessitating it (e.g. W) as the cathode material becomes more refractory.

\section{CONCLUSIONS}

The interactions of a high pressure arc with both refractory and non-refractory cathodes can be described by a unique model. $\mathrm{Cu}$ and $\mathrm{W}$ which are commonly used as cathode materials represent two limiting cases. For $\mathrm{Cu}$, a pressure build-up in the cathode region resulting from the strong vaporization of the cathode surface is necessary for achieving the self-sustaining operation of the arc discharge. Such a pressure buildup is not necessary for $W$. The metallic vapor pressure needed for a self-sustaining operation decreases as the cathode material becomes more refractory.

\section{ACKNOWLEDGMENTS}

The National Sciences and Engineering Council of Canada and the Government of Québec through its FCAR program are gratefully acknowledged for financial support.

\section{REFERENCES}

[1] A. E. Guile, Proc. IEE, IEE Reviews, 1181131 (1971)

[2] T. H. Lee and A. Greenwood, J. Appl. Phys., 32916 (1961)

[3] R. S. Freund, R. C. Wetzel, R. J. Shul and T. R. Hayes, Phys. Rev. A, 413575 (1990)

[4] X. Zhou and J. Heberlein, Plasma Sources Sci. Technol., 3564 (1994)

[5] S. Coulombe and J. -L. Meunier, Plasma Sources Sci. Technol., to be published

[6] S. Coulombe and J. -L. Meunier, J. Phys. D: Appl. Phys., 30 (1997) to be published

[7] B. Jüttner, IEEE Trans. Plasma Sci., PS-15 474 (1987)

[8] J.-L. Meunier and M.G. Drouet, IEEE TPS, PS-15(5) 515 (1987

[9] C.W. Kimblin, J. Appl. Phys., 45(12) 5235 (1974

[10] J.-L. Meunier, IEEE TPS, PS-18(6) 904 (1990)

[11] R. Gayet, C. Harel, T. Josso and H. Jouin, J. Phys. D: Appl. Phys., 293063 (1996)

[12] M. S. Benilov and A. Marotta, J. Phys. D: Appl. Phys., 281869 (1995) 